\title{
A Novel Bat algorithm of solving equation group
}

\author{
Huadan Duoji $i^{1, a}$ \\ ${ }^{1}$ Gansu Normal University for Nationalities, Hezuo, gansu, 747000, China \\ a Huadanduoji@163.com
}

Keywords: Bat Algorithm; Equation groups; Evoluationary computing; Global convergence

\begin{abstract}
To solve equation group problems, this paper constructed a bat algorithm. the algorithm was experimented and the experimental results show that the novel algorithm is effective for solving ill-conditioned linear equation groups.
\end{abstract}

\section{Introduction}

The equations is one of the basic problems in numerical linear algebra, many engineering application and scientific computing problems require the solution of equations finally, therefore, studying the equations has important significance ${ }^{[1]}$. Linear equation group can be divided into the ill-conditioned linear equations and well-conditioned linear equations. The traditional method mainly has the Newton method ${ }^{[2]}$, iterative method, gradient method, evolutionary algorithms such as PSO algorithm ${ }^{[3]}$. The traditional method has high accuracy, but the equations with high performance requirements, such as the requirements of continuous and differentiable equations. Evolutionary algorithm can improve the population size and the number of iterations to improve accuracy, the solving time is proportional to iterative times, time efficiency decreased obviously.

\section{Bat algorithm}

The bat algorithm (BA) is a new heuristic algorithm ${ }^{[4]}$, it is proposed by XinShe Yang in 2010. Yang illustrates the basic idea of this algorithm and puts forward the basic assumption of the bat algorithm:

1) All bats use their echolocation perception and the distance of the target, they identify the target and the different of the background obstacles in a mysterious way.

2) The location of bats are $x_{i}$, flying with the speed of $v_{i}$, they search target at a fixed frequency $f$, variable wavelength $\lambda$ and loudness $A_{i}$. They can determine the distance between himself and the prey and adjust the pulse wavelength (frequency) automatically, at the same time, when they close to the target, they adjust the pulse frequency $f_{i} \in[0,1]$.

3) There are a lot of changes in loudness, the assumption it is from the maximum value of $A_{\max }$ changes to the fixed minimum value of $A_{\text {min }}$.

Since the algorithm is proposed, some scholars have applied this algorithm to solve continuous function optimization problems, and have achieved good results. This paper presents a novel bat algorithm to solve equation group problem, the simulation results show that the algorithm has better convergence speed and precision ${ }^{[5]}$.

\section{The novel bat algorithm}

In order to lead the current optimal bat to produce adaptive variation of the environment, the mutation factor is introduced to design the active evolutionary operator.

According to the mutation factor, we can make the active evolution of the current optimal bats: the position of each bat is defined as a gene. When the evolution probability of this gene $m_{\mathrm{i}}$ is greater than random number, the bit reverse, thus generating an optimal solution of the bat, and compares it with the previous optimal bat, get better as the optimal bat ${ }^{[6]}$. 
If the total weight of a bat represents the selected item exceeds the maximum weight, it is called an invalid bat. For invalid bats, the gene of the bat can be judged according to the calculated mutation factor is 1 or 0 . If the mutation probability $m_{i}$ is less than that of the random number, the gene position is reversed. After the mutation, then determine whether the bat is valid, if it is still an invalid bat, continue to repeat the previous operation, until the bat becomes an effective bat.

In this paper, a new hybrid intelligent optimization algorithm-----genetic variation bat algorithm is proposed. Assuming the bat population was $n$, the position of $i x(i)=\left(\mathrm{x}_{\mathrm{i} 1}, \mathrm{x}_{\mathrm{i} 2}, \ldots \mathrm{x}_{i d}\right)$, where $i=$ $1,2, \ldots n$. The basic steps of genetic variation bat algorithm can be summarized as follows:

The position of $i$ bat in the initial population: $x(i)=$ round $($ rand $(1, d))$, determine each bat of the population is effective, if it is invalid bat, until it becomes effective bat, initial speed $v(i)$, pulse rate $r(i)$, , loudness pulse $A(i)$, pulse frequency $F(i)$ and individual evaluation fitness $(i)=Z(\mathrm{x}(i))$ $(i=1,2, \ldots n)$.

Use the formula $\mathrm{q}_{\mathrm{i}}=a v g{ }_{-}$value $e^{-v c_{i}}$ calculation mutation factor

While (not meeting the end condition)

Use the formula $m_{i}=\left(q_{i}-\min \left\{q_{i}\right\}\right) /\left(\max \left\{q_{i}\right\}-\min \left\{q_{i}\right\}\right)$ calculation mutation factor

for $i=1: n$

Using formula $f_{i}=f_{\min }+\left(f_{\max }-f_{\min }\right) \beta, v_{i}^{t+1}=v_{i}^{t}+\left(x_{i}^{t}-x_{*}\right) f_{i}, x_{i}^{t+1}=x_{i}^{t}+v_{i}^{t+1}$ to generate the new $\mathrm{X}_{\text {new1 }}$

$$
\text { if Rand > R (i) }
$$

Use $A_{i}^{t+1}=\alpha A_{i}^{t}$ to generate the new $\mathrm{x}_{\text {new1 }}$

Endif

Judge the bat whether is effective bat and deal with it

$\mathrm{Z}_{\text {new }}=\mathrm{Z}\left(\mathrm{x}_{\text {new }}\right)$

If rand $<\mathrm{A}(i) \& \& \mathrm{Z}_{\text {new }}>$ fitness $(i)$

$x(i)=x_{\text {new }}$

fitness $(i)=f_{\text {new }}$

Update $R(i)$ and $A(i)$ by $R_{i}^{t+1}=R_{i}^{t} *[1-\exp (-\gamma t)]$.

Endif

Endfor

Update the current best solution $X *$ and its corresponding parameters

Active evolutionary strategy for the current optimal solution

Endwhile

\section{The analysis of simulation}

Run environment of algorithm is MATLAB (R2010b) under Win7 operating system. Parameters: $f_{\min }=0, f_{\max }=1, \alpha=0.95, \gamma=0.9$, the lower bound of speed $v_{\min }=-0.5$, the upper bound of speed $v_{\max }=0.5$, the initial velocity $v=0$, the initial frequency $f=0$, the initial loudness $A=0.5$ and initial pulse frequency $R=0.2$.

Two typical equations are tested. Equations are as follows:

$$
\begin{aligned}
& \text { 1. } \min f_{1}(x)=\sum_{i=1}^{n}\left(x_{i}^{2}-10 \cos \left(2 \pi x_{i}\right)+10\right) \\
& \text { 2. } \min f_{2}(x)=-\frac{\left|\sum_{i=1}^{n} \cos ^{4}\left(x_{i}\right)-2 \prod_{i=1}^{n} \cos ^{2}\left(x_{i}\right)\right|}{\sqrt{\sum_{i=1}^{n} i x_{i}^{2}}}
\end{aligned}
$$


The solution results are as follows:

(a) The use of this algorithm to solve the problem $f_{1}(\mathrm{x})$, the optimal solution is obtained: $[x \min$, $x$ max $]=\left(-1\right.$ 21111900218162E-07, 2.119287120258219E-07), the global minimum value $f_{1}(\mathrm{x})$ $=4.134219256145722 \mathrm{E}-06$. The whole solution process takes $0.27 \mathrm{~h}$. For the optimization problem

$f_{1}(\mathrm{x})$, the use of BA algorithm of Literature [7], when $n=1000$, the optimal solution of $\left[x_{\min }, x_{\max }\right.$. $=[-1.21111900218162 \mathrm{E}-01,2.119287120258219 \mathrm{E}-1]$, the global minimum value $f_{1}(\mathrm{x})=9$. $714917249015812 \mathrm{E}-02$, the whole solution process is $28.73 \mathrm{~h}$.

(b) The use of this algorithm to solve the problem $f_{2}(x)$, When $n=20$, the optimal solution $x$ $=(3.16235390953187,3.12898368752111,3.09155024209814,3.06133201070082,3.0276263796088$ $6,2.99317397448195,2.96057813868317,2.92271131240478,0.495355830406609,0.488664124478$ 412,0.482890733138573,0.476054404941728,0.471321716425919,0.46518220356735,0.46074947 $6025927,0.457126057118075,0.453206871024417,0.449134318069897,0.44350836966006,0.4404$ 05171228925), the global minimum $f_{2}(x)=0.803605881101687$, The whole solution process takes 0.34 h. When $n=30$, the optimal solution $x=6$. 27383767181735,3. 15543063344956,3.12858507579545,3. 10537768619877,3. 08626757890273,3.0738364494704,3. 04421908784382,3. 01625307649929,2. 99613288836597,2.96304309144103,2.95510626490031, 2.92879471689537,0.480793315069097,0.470971408110142,0.464923151131435,0.47169991009 4212,0.452925377244675,0.462755956882183,0.444471092939119,0.458650201100521,0.453005 412757473,0.450369149133667,0.449052963656945,0.457530100747949,0.430066043258168,0.4 43104153112943,0.441285654703739,0.432629271764614,0.42607433502267,0.42260630196341 5 ), Global approximate minimum value $f_{2}(x)=0.821789549623916$, the whole solution process takes $0.47 \mathrm{~h}$. When $n=500$, the global approximate minimum value $f_{2}(\mathrm{x})=0.855219472519328$, the whole solution process is $3.24 \mathrm{~h}$. For the optimization problem $f_{2}(\mathrm{x})$, the use of BA algorithm of Literature [8], when $n=500$, the optimal solution is -0.854185 , But time is not published.

\section{Conclusions}

This paper proposes an improve bat algorithm, and it is applied to equation groups. Due to bat algorithm has the robustness and high computational efficiency, it is suitable for parallel computing, it has great practical value for solving large-scale. Experiments show that: It is feasible and effective to solve equation group.

\section{Acknowledgment}

This work is supported by the Twelfth Five Year Plan of Educational Science in Gansu Province research project [2012](GSGXG039).

\section{References}

[1] David A Cox, John B Little, Donald B. Using Algebric Geometry [M]. New York: Springer Verlag, 1998.

[2] Hentenryck P Van, McAllester D, Kapur D. Solving Polynomial Systems Using a Branch and Prune Approach[J],SIAM Journal on Numerical Analysis, 1997(2):797—927.

[3] Zhao Ji, Xu Wen-bo, Sun Ju. Solving Systems of Nonlinear Equations Using Quantum-behaved Particle Swarm Optimization[J]. Application Research of Compute, 2007(24):80-82.

[4] Xinshe Yang. A new meta heuristic bat-inspired Algorithm [J], Nature Inspired Cooperative Strategies for Optimization (NICSO2010)(Eds. J. R. Gonzalez et al.), SCI 284, 2010:65-74.

[5] Xinshe Yang. Bat algorithm for multi-objective optimization [J]. Bio-Inspired Computation, 2011,3(5):267-274. 
[6] IISUFESCU M. Finite Markov processes and their applications [M] . Wiley: Chichester, 1980 .

[7] YANG Xin-she. A new metaheuristic bat-inspired algorithm [M] / /Nature Inspired Cooperative Strategies for Optimization. Berlin: Springer-Verlag, 2010: 65-74.

[8] Lin Zhiyi, Wang Lingling. An improved self organizing transfer algorithm and its application in Bump problem[J]. Computer engineering and application, 2010, 46 (19): 25-26, 35. 